\title{
Digital transformation and optimization models in the sphere of logistics ${ }^{a}$
}

\author{
Vladimir Anisimov ${ }^{1}$, Evgenii Anisimov ${ }^{2}$, Tatiana Saurenko ${ }^{2 *}$, Artem Smolenskiy $^{2}$ and \\ Oleg Yastrebov ${ }^{2}$ \\ ${ }^{1}$ Peter the Great St. Petersburg Polytechnic University, 195251 Polytechnicheskaya st. 29, Russian \\ Federation \\ ${ }^{2}$ Peoples Friendship University of Russia (RUDN University), 117198 Miklukho-Maklaya st. 6, \\ Russian Federation
}

\begin{abstract}
The efficiency, optimization, speed and time limits have always been of crucial importance for the logistics system, while saving of speed and time in the real-time mode are the key factors with transition to digital technologies and establishment of Industry 4.0 since they become the competitive advantages. The innovative use of technologies in such fields as data analysis, Internet of things and cloud calculations significantly change the logistical and transportation systems as a result of mating digital and existing supply chains becoming the catalyst of transition to "Logistika 4.0". This work offers a model and method of shaping an optimal plan of fulfilling a complex of interrelated logistical operations for such changing conditions. The model is based on the presentation of optimization procedure as a non-linear task of discrete programming consisting in minimization of time of fulfilling the above complex of operations by a limited number of contractors partially interchangeable under conditions of limited budgeting. A model obtained thereat for establishing an optimal plan will belong to the class of nonpolynimially challenging tasks. In order to solve these tasks, a method has been suggested supported by a procedure of branches and boundaries. Thealgorithmisbasedon dichotomous branchingdiagram. Itsapplicationprovidesforreceivingboth quasioptimalandoptimallogisticsplansfor the finite number of steps. Atthat, theassessmentofaccuracy is provided for quasi-optimal plans. The proposed model and method help solve a wide spectrum of practical tasks of logistical planning under conditions of digital transformation.
\end{abstract}

\footnotetext{
aThe publication was prepared with the support of the "RUDN University Program 5-100"

*Corresponding author: tanya@saurenko.ru
} 


\section{Introduction}

The problems of minimizing time of fulfilling a complex of interrelated logistical operations having restrictions for the number of contractors, interchangeability and cost there of make a class of problems widespread in the practice of organization logistical management. Inparticular, theproblemsof industrial production logisticalplanning, development and implementation of innovative projects, planning scientific work in the research establishments and many othersbelong to this class [1-3]. The complexity of such like problems and considerable material and time expenses determined by planning miscalculations bring about the fact that the solution thereof shall be supported not only by the experience and intuition, but by impersonal scientific substantiations too. The applicable mathematical models can be used as tools for such substantiations. The development of structure of such a model and optimization method of the logistical plan is the purpose of this article.

\section{Structure of model of logistical plans optimization}

It is reasonable to reflect composition and interrelation of logistical operations in the form of a network when building models of logistical plan optimization:

$$
H=\{(i, j)\}, \quad i, j=0,1, \ldots, m, \quad i<j,
$$

where, $i, j$ - numbers of network nodes.

Every logistical operation in the network (1) is assigned with an $\operatorname{arch}(i, j)$ connecting $i$ $n$ and $j-n$ nodes. Node $i=0$ corresponds to an event of commencing fulfillment of complex of logistical operations presented by this network. Nodes $i=1,2, \ldots, m$ correspond to the events corresponding to completion of all logistical operations described by the arches included into each of these nodes. The total number of logistical operations (arches) equals $N$.

Thesequenceof logistical operations obeystherule: operation corresponding to an arch outgoing from any node can not be launched before finishing all operations corresponding to the arches included into this node.

Every logistical operation $(i, j)$ is characterized by a required number $n(i, j)$ of operators and duration $\tau(i, j)$.

Let us present a set $K^{*}$ of operators engaged for fulfillment of a complex of logistical operations in the following form:

$$
K^{*}=\{1,2, \cdots, k, \cdots, K\},
$$

where, $k$ - conventional order number (identifier) of operator,

$K$ - number of operators. 
Let us present an interchangeability of operators in the formalized form as the matrix:

$$
\Delta=\left\|\delta_{k}(i, j)\right\|, k=1,2, \cdots, K, \quad(i, j) \in H,
$$

where,

$$
\delta_{k}(i, j)=\left\{\begin{array}{c}
1, \quad \text { if } k-n \text { operator can be engaged in fulfilling } \\
(i, j) \text { logistical operation }, \\
0, \quad k-n \text { operator cannot be engaged in fulfilling } \\
(i, j) \text { logistical operation. }
\end{array}\right.
$$

Let us present the financial expenditures related to engaging operators in fulfilling logistical operations in the form of vector components:

$$
C=\left\|c_{k}(i, j)\right\|, \quad k=1,2, \ldots, K, \quad(i, j) \in H,
$$

where, $c_{k}(i, j)$ - cost of time unit of engaging $k$ - noperator to fulfilling $(i, j)$-n logistical operation.

Thelogisticalplanof fulfilling complex of logistical operations formallycan be presented by a set:

$$
Y=\left\{A_{Y}(i, j), r_{Y}(i, j) \mid(i, j) \in H, r_{Y}(i, j) \subset K^{*}\right\}
$$

where, $A_{Y}(i, j)-Y$ plan - determined moment of time corresponding to beginning of $(i, j)$ - $\mathrm{n}$ logistical operation;

$r_{Y}(i, j)$ - set of operators engaged in fulfilling $(i, j)-\mathrm{n}$ logistical operation according to plan $Y$.

Capacity (number of operators) of set $r_{Y}(i, j)$ equals $n(i, j)$.

Let us suppose that interruption of every launched logistical operation $(i, j) \in H$ is impermissible and the composition $r_{Y}(i, j)$ of a allotted operators will not change until it is completed.

Taking into account the adopted designations, the financial expenses $W_{Y}(i, j)$ for fulfilling logistical operation $(i, j)$ will be calculated according to formula in the course of implementing plan $Y$ :

$$
W_{Y}(i, j)=\sum_{k \in r_{Y}(i, j)} c_{k}(i, j) \tau(i, j)
$$

Let us designate by means of $H_{L}$ a set of all network (1) routes connecting its initial and final vertexes.

Timeoffulfillingtheentirecomplexof logistical operations when implementing plan (5) will be equal to maximum duration $T_{L}$ of route $L \in H_{L}$ from an initial vertex $i=0$ of network (1) to a final one $j=m$. 
Taking into account the adopted designations, the model of forming an optimal plan of fulfilling the complex of logistical operations will take the following form:

Determine logistical plan

$$
Y^{*}=\left\{A_{Y}^{*}(i, j), r_{Y}^{*}(i, j) \quad \mid(i, j) \in H, r_{Y}(i, j) \subseteq K^{*}\right\}
$$

of fulfilling the complex of operations $H=\{(i, j)\}, \quad i, j=0,1, \ldots, m, \quad i<j$, providing fulfillment of condition

$$
T^{*}=T\left(Y^{*}\right)=\min _{Y} \max _{L \in H_{L}} T_{L}(Y)
$$

with restrictions

$$
\begin{gathered}
A_{Y}(i, j) \geq \max _{l, i}\left\{A_{Y}(l, i)+\tau(l, i), \quad(i, j) \in H\right\} ; \\
\sum_{k \in r_{Y}(i, j)} \delta_{k}(i, j)=n(i, j) ; \\
\sum_{(i, j) \in G_{Y}(t)} r_{Y}(i, j) \leq K \\
\sum_{k=1}^{K} \delta_{k}(i, j) \geq n(i, j) \\
\sum_{(i, j) \in H} W_{Y}(i, j) \leq W^{*}
\end{gathered}
$$

where, $G_{Y}(t)$ - set of operations being fulfilled at every moment of time $t$ in case of implementing the logistical plan $Y$;

$W^{*}-$ maximum permissible financial expenditures for fulfilling a complex of logistical operations (1).

Inproblem (7) - (13) condition (8) formalizes a commitment of minimizing time of fulfilling a complex of logistical operations.

Limitation (9) reflectsaruleofintegrating logistical operations identifying that operations outgoing from any network node (1) can begin only aftercompleting all operations included into this node.

Limitation (10) formalizesarequirementof allocation of an established number of operators for every logistical operation.

Limitation (11) reflects a natural planning condition consisting in that the number of operatorsinvited for fulfilling logistical operations simultaneously can not exceed a total number thereof.

Limitation (12) formalizes a requirement consisting in that the number and interchangeability of operatorsshall provide for a possibility of fulfilling every logistical operation of complex (1).

Limitation (13) denotes that total financial expenditures in the course of fulfilling a complex of logistical operations (1) can not exceed an established limiting level. 


\section{Optimization method}

Problem (7) - (13) belongs to the non-linear problems of distributing discrete on-uniform resources in the random network. It is $N P$ - a complex problem of discrete programming [4-6]. Precise methods of solving problems of such class have been offered for the first time in [7-8]. However, renewableresourcesonlyhave been taken into account in the models given consideration in these works. At the same time it is necessary to take into account the necessary non-renewable resources too in the course of planning actual logistical processes in economicalones. In model (7) - (13) the financial resources belong to this type. Relation (13) formallyreflectsthelimitationoftheseresources.

Model (7) - (13) obtained as a result of introducing this relation is a further generalization considered in model [8]. The precise optimization algorithms for model of (7) - (13) type are absent presently. At the same time the demands of practices of planning logistical processes determine a necessity of developing thereof.

For the existence of solution of problem (7) - (13), it is necessary and sufficient that the composition and interchangeability of operators and the established limiting level of $\Omega^{*}$ of financial expenses provide for a possibility of fulfilling all logistical operations of complex (1).

In the formalized form the fulfillment of the first of these requirements consists in fulfilling limitation (12).

In order to check fulfillment of the second requirement, one can use the following set:

$R^{*}=\left\{r^{q}(i, j) \mid(i, j) \in H, q=1,2, \ldots\right\}$ of all possible variants of allotting resources for the applicable logistical operations. The following set is connected with this set:

$$
\hat{W}=\left\{W^{q}(i, j), \quad q=1,2, \ldots, \quad(i, j) \in H\right\}
$$

Of financial expenses $\Omega^{q}(i, j)$ for fulfilling logistical operations with the corresponding variants of engaging operators. The elements of set (14) will be determined by the relation:

$$
W^{q}(i, j)=\tau(i, j) \sum_{k \in r^{q}(i, j)} c_{k}(i, j) .
$$

Taking into account (15) these condrequirement providing for existence of a solution of problem under consideration $(7)-(13)$ is presented in the formalized form by the relation:

$$
\sum_{(i, j) \in H} \min _{q} W^{q}(i, j) \leq W^{*}, \quad q=1,2, \ldots .
$$

It is reasonable to check practicability of conditions (12) and (16) evenbeforecommencementofformationprocedurewith the use of model (7) - (13) of optimal plan of fulfilling a complex of interrelated logistical operations. If these conditions are met,an algorithm including the following actions can be used for optimizing a plan of fulfilling a complex of logistical operations (1):

a) presentation of $\operatorname{set} V=\{S\}$ of fragments $S$ of logistical plan $Y$ permissible with respect to limitationsin the form of a tree of subsets (branching);

b) calculating tree branches (dedicated subsets) lower boundary of target function (8);

c) search for permissible variants of logistical plan;

d) checking established permissible variants for optimality.

Thebranchingofatreeofvariantsis effected on the basis of dichotomous diagram. In case of implementing it every vertex of $v_{s} S-\mathrm{n}$ branch of a tree of variants corresponds to an 
element of logistical plan. Inthiscase, ifthe logistical operation $(i, j)$ corresponding to this element begins at the moment of time $A_{S}(i, j)$ a $t_{S}(i, j)$-n variant of assigning operators, we suppose:

$$
v_{S}=\left\{A_{S}(i, j), r_{S}(i, j)\right\} .
$$

Ifthe logistical operation $(i, j)$ does not begin at the moment of time $A_{S}(i, j)$ a $\operatorname{tr} r_{S}(i, j)$-m variant of assigning operators, we suppose:

$$
v_{S}=\varnothing \text {. }
$$

For every branch $S \in V$ values $A_{S}(i, j),(i, j) \in H$, (moments of beginning logistical operations) shall be selected from an increasing sequence corresponding to this branch:

$$
t_{S}=\left\{t_{S}^{n}\right\}, \quad n=1,2, \cdots .
$$

In this case, $t_{S}^{1}=0$, while the subsequent moments $t_{S}^{n}, \quad n=2,3, \cdots$, will be determined on the basis ofinterrelation:

$$
t_{s}^{n}=\min _{(i, j) \in G\left(t_{s}^{n-1}\right)}\left\{A_{s}(i, j)+\tau(i, j)\right\},
$$

where, $G_{S}\left(t_{S}^{n-1}\right)$ - set of logistical operations $(i, j)$, include dearlier into $S$-n branch and incomplete by the moment of time $t_{s}^{n-1}$, i.e.:

$$
G_{S}\left(t_{S}^{n-1}\right)=\left\{(i, j) \mid(i, j) \in H, A_{S}(i, j) \leq t_{S}^{n-1} \leq A_{S}(i, j)+\tau(i, j)\right\} .
$$

Thus, $t_{s}$ corresponds to a sequence of time moments, in to which logistical operations included into the next tree branchare completed and the corresponding operators get released.

Condition $t_{S}^{1}=0$ reflects a fact that all the variants of a logistical plan begin at the moment of time $t=0$.

In the interests of implementing the adopteddichotomousdiagramofbranchinglet us introduce a set for consideration:

$$
D=\left\{d_{q} \mid q=1,2, \cdots\right\}
$$

where, $d_{q}=1$, if for fulfilling $(i, j)$-n logistical operation a variant $r^{q}(i, j)$ of assigning operators is used, otherwise $d_{q}=0$.

In this case the order number $q$ of element $d_{q}=1$ of set $D$ characterizes both fulfilled logistical operation and a variant of appointing operators, and financial expenses.

Taking into account (21) the process of branching in the interests of compiling an optimal logistical plan (7) consists in selecting permissible variables $d_{q} \in D$ for every following moment of time $t_{s}^{n}$ and setting values thereof, i.e., an interrelation takes place $v_{S}=\left\{t_{s}^{n}, d_{q}=1\right\}$, if the logistical operation $(i, j) \in G$ corresponding to a variable $d_{q}$ begins at the moment of time $A_{S}(i, j)=t_{S}^{n}$ at $r^{q}(i, j)$-n variant of allotting resources or $v_{S}=\left\{t_{S}^{n}, d_{q}=0\right\}$, if the specified logistical operation does not begin during a variant of allotting resources under consideration at moment $t_{S}^{n}$. 
Set $P_{S}^{n}$ of variables $d_{q} \in D$, which can be included into $S-n$ fragment of the logistical plan (7) at the moment of time $t_{S}^{n}$, comprises values $d_{q} \in D$ corresponding to logistical operations meeting conditions $(i, j) \in G$, not included earlier into branch $S$ under consideration:

$$
\begin{gathered}
A(l, i)+\tau(l, i) \leq t_{s}^{n}, \quad(l, i) \in H ; \\
r^{q}(i, j) \subseteq R_{S}^{n}, \\
W^{*}-\sum_{d_{m} \in D_{s}\left(t_{s}^{n-1}\right)} d_{m} W^{m}+W^{q} \geq 0, \quad q=1,2, \ldots,
\end{gathered}
$$

where, $R_{S}^{n}$ - set of free operators for $S$-nplan fragment at the moment of time $t_{S}^{n}$;

$D_{S}\left(\begin{array}{c}t_{S}^{n-1} \\ )\end{array}\right)$ - set of variables $d_{q}$ included into branch $S$ of the tree of variants by the moment of time $t_{s}^{n-1}$.

Condition (22) distinguishes logistical operations, for which all the preceding conditions have been met by the moment $t_{s}^{n}$. Condition (23) distinguishes logistical operations, for which there exist permissible job orders of operators, while (24) - permissible job orders of free operators permissible with respect to financial expenses.

A maximum route duration from the initial vertex of graph $H$ to a final one being determined without taking into account life restrictions (11), (13) for logistical operations not included into $S$ can be taken for evaluating $Q_{S}$ of the lower boundary of target function (8) for every fragment $S$ of calendar plan. At that, if at the next branching step corresponding to moment $t_{s}^{n}, d_{q}=1$, is established, in order to determine $W_{S}\left(d_{q}=1\right)$ the following proceeds:

a) logistical operations $(l, i) \in H$, include dearlier into $S$-n plan fragment (i.e., operations, for which $\left.A_{S}(l, i)<t_{S} n\right)$ begin at the corresponding moments $A_{S}(l, i)$ and come to an end at moments $A_{S}(l, i)+\tau(l, i)$;

b) for logistical operation $(i, j) \in H$, corresponding to variable $d_{q}=P_{S}^{n}$ and, hence, included at the step under consideration into $S$-n branch of variants tree $-A_{S}(i, j)=t_{S}^{n}$;

c) for logistical operations $(e, h) \in H$, corresponding to variables $d_{u} \in P_{S}^{n}, \quad u \neq q$, which with respect to life restriction (24) can not be included into plan at the moment $t_{s}^{n}$ simultaneously with $(i, j)$, commencement time equals $t_{s}^{n+1}$, while duration is determined by the relation $t_{S}^{n+1}+\tau(e, h)$.

If $d_{q}=0$, is established at the considered branching step, in order to determine $Q_{s}\left(d_{q}=0\right)$ the following is due additionally:

a) logistical operations $(l, i) \in G$, include dearlier into $S$-n fragment of the logistical plan (i.e., operations, for which $\left.A_{S}(l, i)<t_{S}^{n}\right)$, begin during corresponding moments $A_{S}(l, i)$ and come to an end at moments $A_{S}(l, i)+\tau(l, i)$; 
b) logistical operation $(i, j)$, corresponding to a variable $d_{q} \in P_{S}^{n}$, begins at the moment of time $t_{S}^{n+1}$ and comes to an end at moment $t_{S}^{n+1}+\tau(l, i)$;

c) the rest logistical operations $(e, h) \in G$ corresponding to variables $d_{u} \in P_{S}^{n}, \quad u \neq q$, begin at moment $t_{S}^{n}$ and come to an end at moments $t_{s}^{n}+\tau(e, h)$.

Animportantelementof optimization algorithm under consideration for model(7) - (13) significantly influencingits reproducibility is the method of selecting the nextlogistical operation and variant of allotting resources for it. Formally, it consists in selection of variables $d_{q} \in P_{s}^{n}$ to be included into $S$-n branch at the moment of time $t_{s}^{n}$. Selection of $d_{q}$ will be effected in the offered algorithm at the next branching step in two stages: logistical operationis selected at the first one, and a variant of allotting resources is selected at the second one.Thenext logistical operation will be selected in accordance with the following sequence of preferences:

$$
\min T_{j}^{(n)} \rightarrow \max \tau(i, j) \rightarrow \min i \rightarrow \min j,
$$

i. e. logistical operation is the first to be included into plan with the less late completion time $T_{j}^{(n)}$ corresponding to it. In case there are several such logistical operations an operation of maximum volume will be chosen from this number. In case there are also several such logistical operations an operationwith the least numbers $i, j$ will be chosen. At that, later completion periods are to be determined taking into account fragment $S$ of the logistical plan under consideration.

Variant $r(i, j)$ of assigning operators for the selected logistical operation $(i, j)$ will be determined proceeding from the condition of value minimum:

$$
\sum_{k \in r(i, j)} \sum_{(l, h) \notin S} \delta_{k}(l, h)
$$

i.e., the least universal operators get assigned for the remaining logistical operations $(l, h) \notin S$.

The logistical operation $(i, j)$ selected in this way and variant $r(i, j)$ of assigning operators unanimously determine the next variable $d_{q} \in P_{S}^{n}$, included into $S$-n branch of plan variants tree at the moment $t_{s}^{n}$.

Thetreegoing around is organized in accordance with "go right" rule. Ithelpskeepthe current fragment of the logistical plan, the least value of target function received earlier and a permissible plan corresponding to it in PC memory when solving a problem.

The indicated rule in combination with the considered method of selecting logistical operations and operators constitutes an approximate algorithm of logistical plan optimization, which helps acquire the first permissible solution over the finite number of steps equal to number $N$ of logistical operations in network (1).

Every $S$-n branch comes to an end, if it has included all $N$ logistical operations, i.e., a permissible logistical plan $Y$, was received or if

$$
Q_{s} \geq T^{0}(1-\mu), \quad 0 \leq \mu \leq 1,
$$

where, $T^{0}$ - the least value of target unction for permissible logistical plans received earlier; 
$\mu$-specified permissible target function deviation from an optimal one (optimization accuracy).

Fulfilling condition (26) means that it is impossible to improve a record received earlier by more than $100 \mu \%$ in the branch being considered and its continuation in the framework of a specified accuracy of optimization has no sense.

A procedure of searching solution comes to an end, if condition (26) is met for all the remaining branches. In case of an established method of going around a tree of variants the second return to the root vertex corresponds to such a situation. In this case the last record is the sought value of target function (8), while a permissible logistical plan corresponding to it-an optimal plan $Y^{*}$.

In general the offered model and method of planning logistical processes provide for an automatic viewing and assessing all possible plan variants and receiving on this basis both accurate and approximate solutions of the planning problem. In this case repetitions are excluded in the course of viewing permissible variants. It significantly reduces the time of solving problems.

\section{Conclusion}

The offered model and method can be used in the systems of supporting decisions-making used in the practice of logistical management of organizations. They help formalize complexes of logistical operations different by the nature thereof. In this case the system approach to forming a plan of logistical process, reviewing it as a unified non-separable complex of interrelated logistical operations and the life support thereof aimed at attaining general final goals, make it possible to reduce material and time expenses of the participants of logistical process, reduce time limits of resources delivery and increase the quality of servicing.

\section{References}

1. G.Y. Silkina, Proc. of the 29th IBIMA Conf. - Sustainable Economic Growth, Education Excellence, and Innovation Management through Vision 2020, (2017)

2. A.S. Ksenofontov, I.V. Savon, V.Y. Serba, D.V. Shkurkin, International Journal of Economics and Financial Issues, 6(S1) (2016)

3. V.G. Anisimov, E.G. Anisimov, T.N. Saurenko, M.A. Sonkin, Journal of Physics: Conference Series, 803 (2017)

4. A. O. Alekseyev, O. G. Alekseyev, V. G. Anisimov, Ye. G. Anisimov, N. I. Yachkula, Soviet Journal of Computer and Systems Sciences, 5(1988)

5. M. D. Maslakov, A. K. Chernykh, Risk Management Problems in Technosphere, 1 (29) (2014)

6. V. G. Khalin, G. V. Chernova, A. V. Yurkov, Economical Analysis: Theory and Practice, 7 (406) (2015)

7. V.G. Anisimov, Ye. G. Anisimov, The Magazine of Computational Mathematics and Mathematical Physics., 32 (2) (1992)

8. V.G. Anisimov, E.G. Anisimov, The Magazine of Computational Mathematics and Mathematical Physics., 37(1) (1997) 